\title{
Online Persuasion in Facebook and Mixi: A Cross-Cultural Comparison
}

\author{
B.J. Fogg and Daisuke Iizawa \\ Stanford University \\ Cordura 122, Stanford, CA 94305 \\ bjfoggastanford.edu \\ daisuke.iizawa@gmail.com
}

\begin{abstract}
Social networking sites persuade millions of users each day to adopt specific behaviors. To understand this phenomenon in the context of persuasive technology, we analyzed how persuasion takes place in leading social networking sites from two different countries: Facebook in the U.S. and Mixi in Japan. We compared the two services on four persuasion goals: creating profile pages, inviting friends, responding to content by friends, and returning to the site often. Our analysis reveals the differences and similarities in how Facebook and Mixi are designed to influence users toward the achievement of these four goals. In general, Facebook's persuasive design is more assertive and mechanistic, while Mixi's approach, by comparison, is subtle and indirect. These persuasion styles seem to map generally to cultural differences between the U.S. and Japan.
\end{abstract}

Keywords: persuasion, captology, social networking, persuasive technology, behavior chain, persuasive design, cultural comparison, Mixi, Facebook.

\section{Introduction}

Social networking services are among the most popular sites on today's Internet. They are also among the most persuasive. For a social networking service (SNS) to succeed, the service must motivate users to adopt specific target behaviors: register, upload a photo, connect to friends, share content with friends, and so on. The study of social networking sites can provide insight into how persuasion occurs online, an increasingly important topic in captology, the study of persuasive technology [1].

Facebook is the fastest growing SNS in the U.S., ranking \#5 in traffic ${ }^{1}$ for all U.S. websites. However, in Japan Facebook is not popular, failing to rank in the top 100 sites. Instead, Mixi is the leading SNS, ranked as the \#5 website overall in Japan².

Despite the profound cultural differences between the U.S. and Japan, people in both countries are being motivated and persuaded on a daily basis by these social networking services. As a research team from the U.S. and Japan, we set out to compare how persuasion occurs in Facebook and Mixi by analyzing the user

${ }^{1}$ Data from Alexa.com on January 1, 2008.

${ }^{2}$ Data from Alexa.com on January 1, 2008. 
experiences from the perspective of persuasive technology. To our knowledge, our research is the first collaborative effort between countries to compare the persuasive design of social networking services that appeal to two different cultures.

Our comparison is a case study. As such, we do not attempt to test hypotheses. Instead, our goal is to illuminate which issues deserve deeper investigation and which could later become independent variables in a controlled experiment.

Our work makes an original contribution by specifying persuasive goals in SNSs and describing how two services achieve these goals; comparing influence strategies used in online settings from two different cultures; and analyzing the success of SNSs in terms of persuasive technology principles.

\subsection{Background}

Fogg \& Eckles [2] investigated over 50 successful "Web 2.0" services and identified a pattern that all leading services followed, which they called the "Behavior Chain for Online Participation." This framework, illustrated in Fig. 1, was a useful lens through which to compare persuasion on Facebook and Mixi. The Behavior Chain outlines three Phases: Discovery, Superficial Involvement, and True Commitment. Our comparison of Facebook and Mixi focuses on Phase 3: True Commitment.

Our analysis revealed that both Facebook and Mixi are designed to persuade users toward the behaviors listed in Phase 3: creating value and content, involving others, and staying active and loyal. To focus our research, we extracted four SNS persuasion

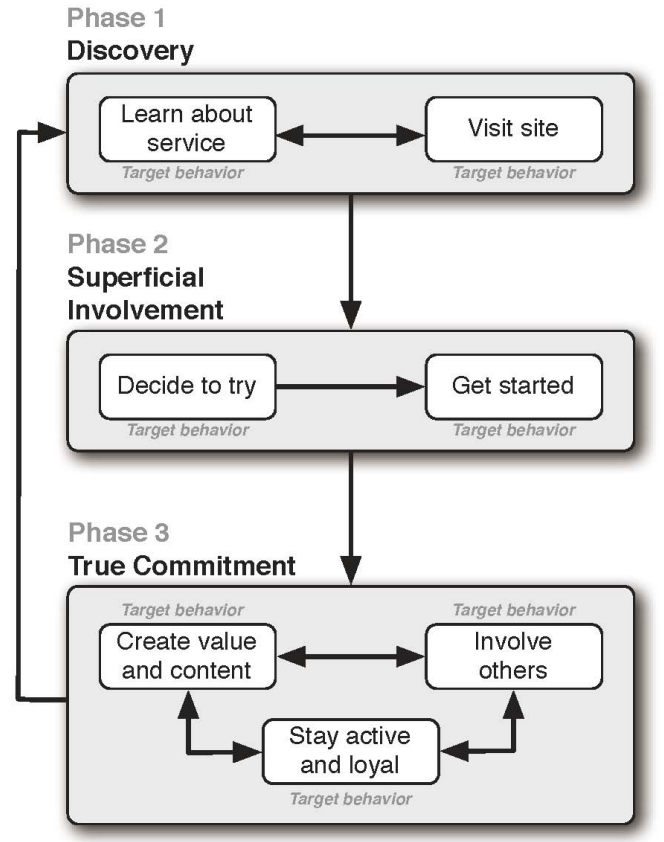

Fig. 1. Our cross-cultural analysis focuses on Phase 3 of the Behavior Chain [2] 
goals that map onto the Target Behaviors listed in Figure 1. These persuasion goals are generally accepted objectives for a commercial site. Below we list our SNS persuasion goals and show how each corresponds with a Target Behaviors from the Behavior Chain.

\section{Persuasion Goals for SNS}

\#1 Create Personal Profile Page

\#2 Invite Friends

\#3 Respond to Others' Contributions \#4 Return to Site Often

\section{Target Behaviors in Behavior Chain}

$-->$ "Create Value and Content"

$->$ "Involve Others"

$-->$ "Create Value and Content"

$-->$ "Stay Active and Loyal"

In this paper we examine how Facebook and Mixi are designed to achieve the four persuasion goals listed above. In the sections that follow, we explain each goal and the persuasive design in Facebook and Mixi to achieve that goal. Then we briefly compare and contrast the persuasion strategies. We conclude our paper with a summary and an overview of issues for future investigation.

\section{Comparison of Persuasive Design Elements}

The commercial success of Social Networking Services depends on persuading users to perform specific behaviors. The persuasion goals we discuss in this paper are among the most important goals for commercial viability. If a SNS fails to achieve any one of these four goals, the company will likely fail. For this reason, these four goals are the focus as we compare Facebook and Mixi.

\subsection{Persuasion Goal \#1 - Create Personal Profile Pages}

Profile pages form the core of social networking sites. The quality of experience on these sites is tied to the quality of the profile pages. We found that both Facebook and Mixi are designed to motivate users to create engaging profile pages, though each service takes a different approach.

On Facebook, the "edit" command for the profile page is always visible to the user. This visual prominence serves two functions. First, the interface reduces the barrier [3] for adding information. Next, the design implies that a profile page can and should be updated frequently.

As shown in Figure 2, the Facebook interface provides users with a series of labeled tabs, each with drop-down boxes or free-response spaces. To induce people to create interesting profile pages, the Facebook interface prompts users for highly personal information, such as religious views, mobile phone number, sexual orientation, and relationship status. Facebook does not indicate that such responses are optional.

Mixi also prompts users to share and update personal information. However, Mixi divides the initial profile creation experience into two stages. In addition to verifying the user's email address, this two-stage model serves two persuasive purposes: It builds self-efficacy [4] for using Mixi, and it leverages a "foot in the door" approach 


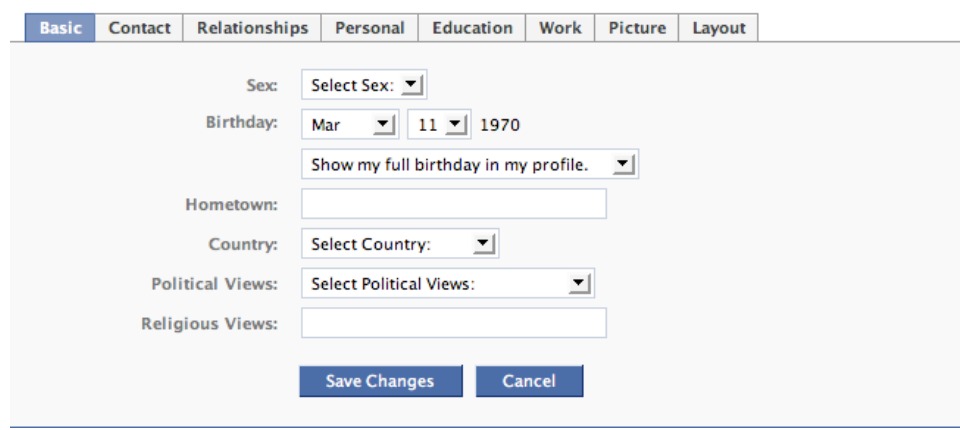

Fig. 2. The Facebook user interface prompts users for highly personal information such as political, religious, and sexual orientation

to persuading the user to offer future disclosures $[5,6]$. In the first stage, the user enters a name and a "self-introduction." The Mixi interface models friendly behavior [7] by offering users a sample self-introduction, which we've translated as follows:

"Hello, my name is Mixi Tanaka. I am a college student. I would like to be a counselor to help people. I am wondering if I can communicate with your friends in Mixi. I'm looking forward to meeting you on Mixi."

The design of the Mixi interface, shown in Figure 2, visually suggests the length and tenor of the new user's self-introduction. In this way, Mixi likely influences users toward friendly and concise self-introductions.

After submitting the self-introduction, Mixi users have completed the first stage of profile creation, likely giving new users a sense of success and, equally important, setting a precedent for further disclosures in the second stage. Users will share the bulk of their profile information in the second stage. This includes hobbies and personal interests. Mixi does not prompt for highly personal information.

While both Facebook and Mixi are designed to elicit personal information, the services differ in two ways. First of all, Mixi's two-stage model allows users to build confidence and begin slowly to disclose information. If the first stage of the model can establish trust, users will likely reveal more information in the second stage [8].

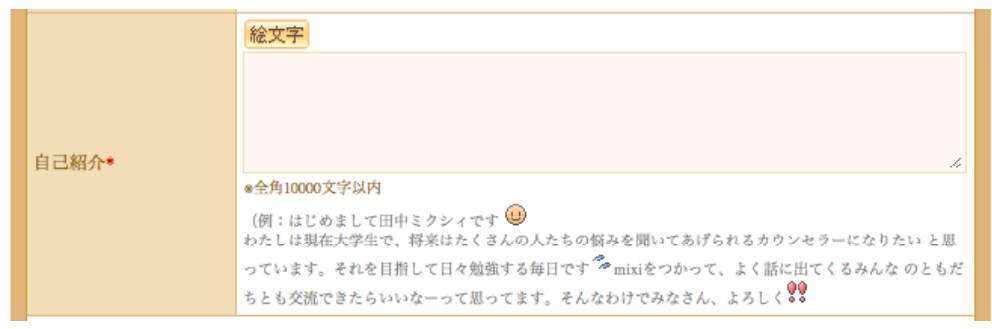

Fig. 3. The Mixi UI models behavior for creating a friendly self-introduction 
In contrast, the Facebook profile creation process is one long stage, with no initial trust-building period. The Facebook interface prompts users for highly revealing information, while Mixi's interface does not.

In both services, users are encouraged to change and update their profile by an interface design that makes such behavior simple.

\subsection{Persuasion Goal \#2 - Invite Friends}

Inviting and connecting to friends is an essential behavior for making a SNS successful. Without these connections, the service would have little value. The design of Facebook and Mixi both persuade people toward achieving this key outcome.

Facebook takes bold measures to motivate users to invite others to the service. Users can click on the always-present "Friends" interface element to invite individuals by typing in their email addresses. But the persuasion tactics of Facebook do not stop with visual prominence and simplicity. Facebook also offers tools that allow users to invite friends in bulk.

One such invitation tool, shown in Figure 4, gives Facebook access to the user's password-protected contact list on other services, such as Yahoo, AOL, or Gmail. The Facebook interface conveys simplicity and credibility to gain user compliance (The UI says, "Facebook will not store your email login or password."). After the user enters confidential information, the Facebook technology logs in to the competing service such as Yahoo, extracts the user's contacts, and sends invitations to everyone the user designates. The bulk invitation tool allows new users to have hundreds of Facebook friends quickly - a strong incentive in Facebook's culture where having friends is a mark of status [8]. The bulk invitation process takes about two minutes.

Compared to Facebook, Mixi takes a less aggressive approach to motivating invitations. Like Facebook, the invitation tool is easy to access, reducing barriers to achieving this persuasive goal. However, in contrast to Facebook's automation, Mixi users must type in the email address for each friend they wish to invite. There is no tool for bulk invitations. As a result, connecting with friends in Mixi requires effort for each individual invited.
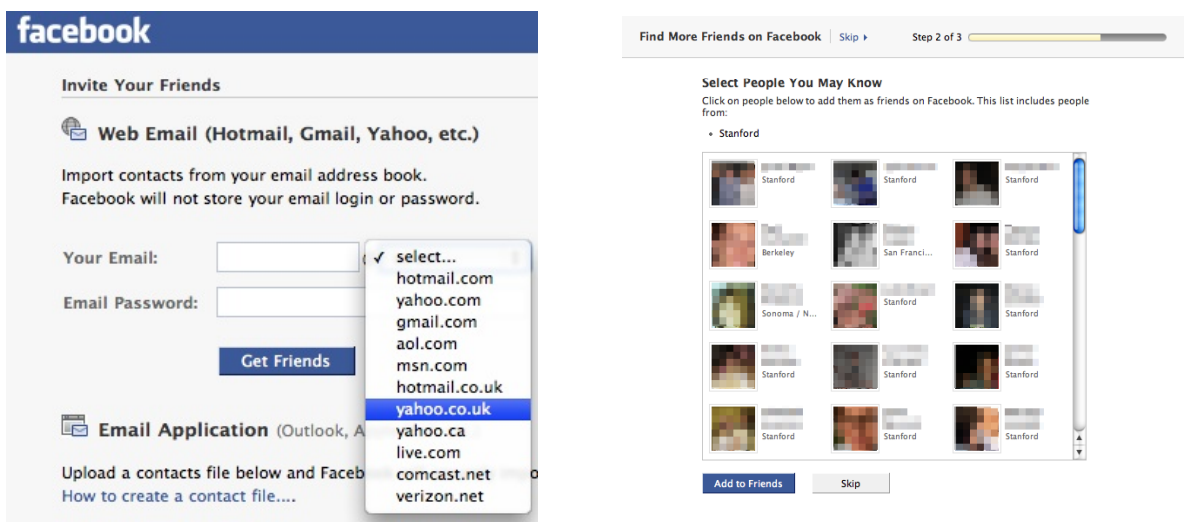

Fig. 4. Facebook encourages users to invite friends in bulk, often hundreds of people at once 


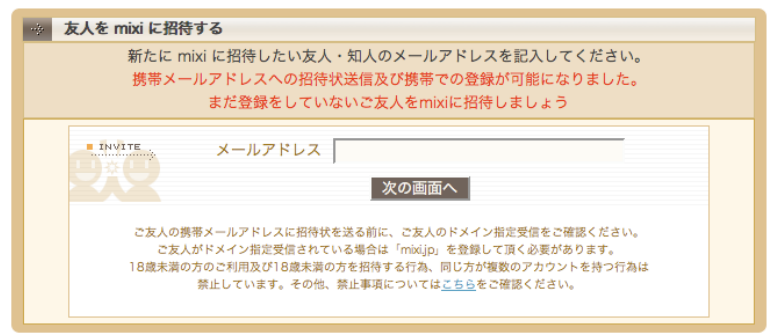

Fig. 5. Mixi users are encouraged to invite friends, but users cannot invite in bulk - only one person at a time

Both Facebook and Mixi motivate users to connect to more friends by publicizing the number of friendship connections for each user.Both services allow other people to see if a user has one friend or 500. Having only a few friends seems to be a marker of low status in both Mixi and Facebook. So users naturally have a drive to add friends in order to be seen as more socially desirable.

In Mixi, the number of friendship connections is linked to each user's name on the Friends List, as shown in Figure 6. The social connectedness of friends is easily compared. For example, Figure 6 shows one Mixi user with 200 friends, while other people have only one friend. Social comparison and social desirability factors naturally motivate users to build their friendship network.

In contrast with Mixi, Facebook doesn't offer an interface that shows all the friendship numbers in one place. Rather, the number of friends is found on each person's profile page. As shown in Figure 7, Facebook organizes friendships by "Network," which is an artifact from Facebook's early days as a series of college directories or networks. (The early design did not allow networks to exchange data.)

To this point in our analysis, we noted that Facebook has consistently motivated users with persuasive design that is more assertive and direct than Mixi's design. However, in this single instance-the way services convey social statistics-Mixi's design is more assertive. Mixi connects the friendship numbers directly to the user's

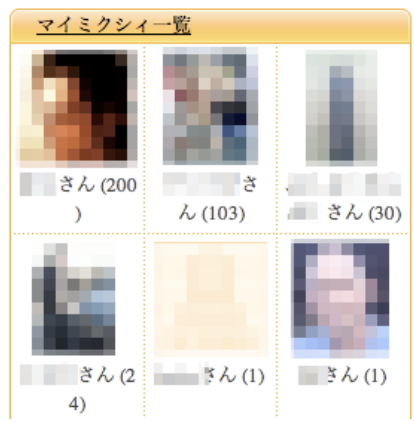

Fig. 6. Mixi publicizes the number of friends for each user, which creates motivation for connections. In this example, one user has 200 connections while others have just one. 

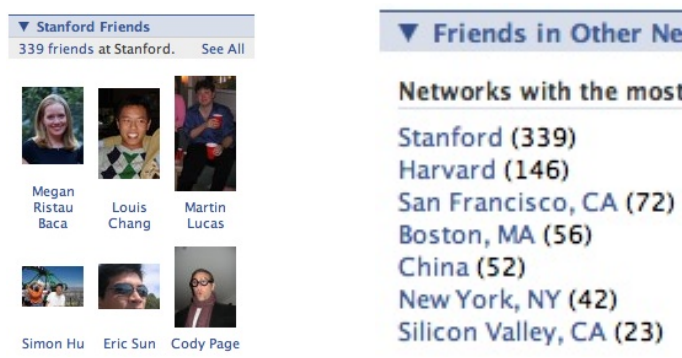

Fig. 7. Facebook publicizes the quantity of connection for each user, organized by Network

name, making this data part of the user's online identity. In addition, Mixi makes it easy to see which friends have the most connections. In contrast, to compare friends in this way on Facebook would take significant work.

In our research comparing Mixi and Facebook we speculated that the motivation to acquire many online friends may be stronger in the U.S. than in Japan. More specifically, on Facebook the drive to have many friends is an accepted part of the online experience [9]. We could find no prior research on this point so we set out to gather data as a pilot test of our hypothesis about the desirable number of social connections.

We performed a small online survey in Japan and the U.S., using a convenience sample of users from Mixi $(n=34)$ and Facebook $(n=30)$. The results, while not definitive, suggest that each culture has a different view of how many social connections online is ideal, at least on Facebook and Mixi.

The survey summary in Table 1 shows that Mixi users in our sample had substantially fewer online connections, with an average of 58 friends compared to an average of 281 friends for Facebook users.

Table 1. Facebook users are motivated to connect with more friends than Mixi users

\begin{tabular}{lcc}
\hline Question in Survey & Facebook Users & Mixi Users \\
\hline Current number of friends in SNS (average) & 281 & 58 \\
Hoping to add more friends online & $23 \%$ & $9 \%$ \\
"Ideal" number of friends in SNS (average) & 317 & 49
\end{tabular}

The survey showed that users of the two services also perceived the "ideal" number of SNS friends differently, with Mixi users choosing 49 connections on average as ideal, while Facebook users reported an average of 317 connections as ideal. Similarly, when asked if they wanted to add more friends online, $23 \%$ of Facebook users said "yes," while only $9 \%$ of Mixi users hoped to add more friends.

Figure 8 maps the survey data to highlight the differing views (and therefore motivation strength) regarding the quantity of online social connectedness. The plot 


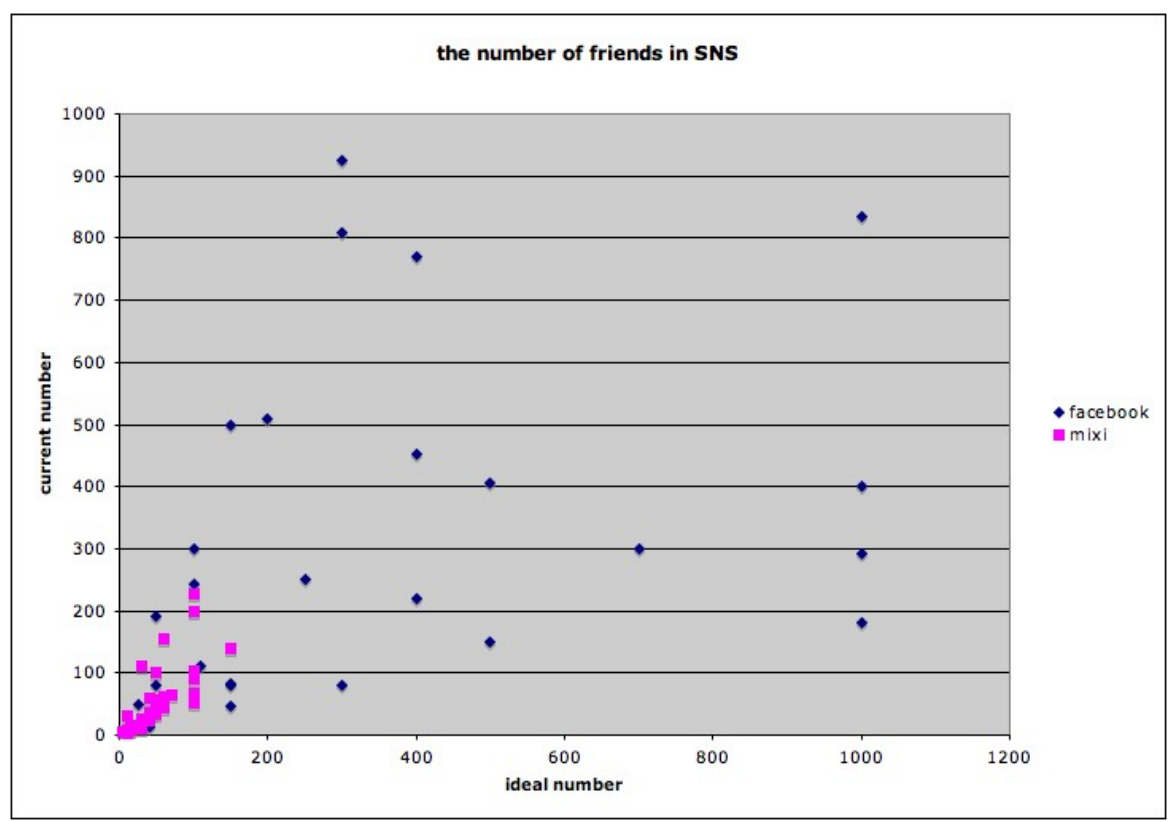

Fig. 8. Mixi users are connected to fewer friends than Facebook users. Compared to Mixi users, Facebook users aspire to have more connections, sometimes up to 1000.

shows a tight cluster for Mixi users in the lower left-hand quadrant of the graph. Compared to Facebook users, Mixi users generally have fewer connections in Mixi, and they seek fewer connections.

The apparent difference in how Mixi and Facebook users perceive the quantity of social connections suggests that Facebook can leverage the motivation to make more connections than can Mixi in designing for persuasion. Some might propose that Mixi should copy Facebook's approach. This could be a mistake. For Japanese users, Facebook's "mass invitation" tool may seem crass and insincere. In contrast, for a U.S. user of Facebook, mass invitations may appear to be a smart way to save time.

This difference in quantity of relationships also seems to match the cultural differences in how people manage social relationships in the U.S. and Japan [10, 11]. The persuasive design in Mixi and Facebook reflect (and perhaps reinforce) those differences. Our specific conclusion matches a larger pattern of persuasive technology tailored to the motivational characteristics of various cultures [12].

\subsection{Persuasion Goal \#3 - Respond to Others' Contributions}

People gain satisfaction from posting content online, but they likely get more satisfaction from seeing others responding positively to what they have posted. Because social interaction over user-generated content is what sets SNSs apart from traditional media and web sites, our third comparison between Facebook and Mixi examines how these services motivate users to view and comment on their friends' content. 
Facebook and Mixi allow users to post content in video, text, links, and photos, and both services notify users about new content posted by friends. This serves as the first step toward initiating a response to other people's content.

Facebook notifies friends about new content in two ways. First, the Facebook News Feed, which occupies the central part of each user's "Home" page, shows friends' recent activities, including newly posted content. Users can see the content with one click, a barrier reduction strategy that motivates this behavior.

Next, in Facebook any posting of new comments also generates an email to the user's email address outside of Facebook. This again makes the content one click away, but this time the notification is pushed to the user's email, expanding Facebook's reach beyond the web site. Similarly, Mixi notifies users about new content via email. For both services, the email notification is a first step in getting people to engage with user-generated content.

Both Facebook and Mixi encourage people to comment on what friends have posted online. The services make this easy to do by placing a prominent comment box near the posted item. The interfaces are similar, as shown in Figure 9. The design in both cases conveys to users that posting a comment is simple: an open field for entry plus a simple submit button. The size of the comment box implies brevity, which makes it easier for others to read the comment later. Indeed, the design of the commenting interfaces likely influences the desired behavior among friends.
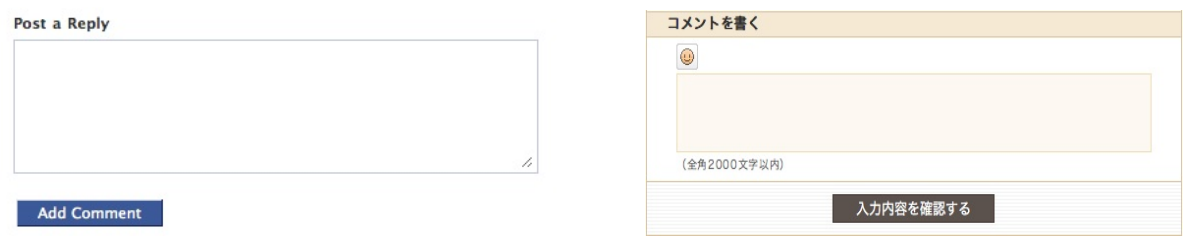

Fig. 9. The UIs for Facebook (left) and Mixi (right) encourage commenting about user content

The user experiences for commenting are similar in Facebook and Mixi. In both services, multiple comments on one piece of content become a sort of dialogue, allowing a variety of friends to react and discuss together.

In terms of persuading users to respond to the content friends have posted, Facebook and Mixi are identical from a psychological perspective. Both services trigger user behavior by sending an email notification, and both reduce barriers by including links in email and then making the commenting box prominent. This similarity is not surprising. Motivating friends to interact online about shared content is the "holy grail" of social networking. In our view, more than any other activity, the experience of interacting with shared content motivates people to continue using Facebook and Mixi.

\subsection{Persuasion Goal \#4 - Return to Site Often}

A goal of all social networking services is to persuade users to return often. Active users make the service more engaging for others in their online social network. In our research, we identified and compared three ways that Facebook and Mixi motivate 
users to return to the site often: through email updates, digests of friends' activities, and "pokes" (Facebook) or "ashiato" (Mixi).

Persuading Returns via Email Updates. Both Mixi and Facebook send notices to users' email accounts to update them on new happenings with their friends in the SNS. Mixi sends a daily update, which has only the titles of the newly posted content. To see more than the titles, users must click on a link and log into the service.

Facebook's notification system is similar but more assertive. Facebook sends users an email notification when any of the following happens: when users are tagged in a posted content item, have comments posted about them, have comments posted subsequent to their own, receive a message on Facebook, are added as a friend, or are requested to join a group. As a result, a Facebook user could receive dozens of emails a day from the SNS. Each email is designed to motivate users to return to the site.

In comparing these two approaches, we find the Mixi solution to be less direct and, in some ways, more graceful. The Facebook notifications are direct and, thanks to the volume, potentially more annoying.

Persuading Returns with Digest of Friends' Activities. The second way to compare how these services motivate return visits is to examine how users stay updated on their friends' activities. In this regard, the sites are psychologically similar. Users of both systems can view friends' activities in one place on each site. In Facebook, the News Feed recounts who has posted a new link, who has signed up for an event, who is in a new relationship, and so on. On Mixi, the content is less diverse but the function is the same. Mixi shows updates on the Diary, on photos, and more. The drive to stay up-todate with friends' activities [13] likely prompts frequent return visits.

Persuading Returns with Pokes and Ashiato. The third way we compare the persuasive design for return visits is identifying how users are socially present in each others' online spaces. On Facebook, users can visit a friend's profile page and then send that friend a "poke." The ambiguous "poke" is sent via email and SMS to the friend. Poking can prompt a user to log in and "poke" back - or to send a message, write on their Wall, or respond in other ways. By design, "poking" is a provocation.

In contrast, Mixi doesn't offer "poking." Instead, one user becomes aware of another's presence on his or her profile page by "ashiato," which is translated as "footprints." When a user logs into Mixi, she can see evidence that other users have visited her profile page by viewing the Access Log and the accompanying footprints, as shown in Figure 11.

\section{Pokes \\ You were poked by Alison. poke back | hide poke}

Fig. 10. Facebook users can "poke" friends when visiting their profile pages 


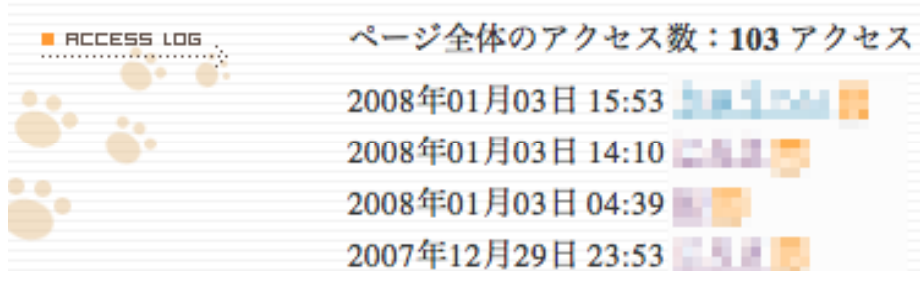

Fig. 11. Mixi shows who has visited a friend's profile page by leaving "Footprints"
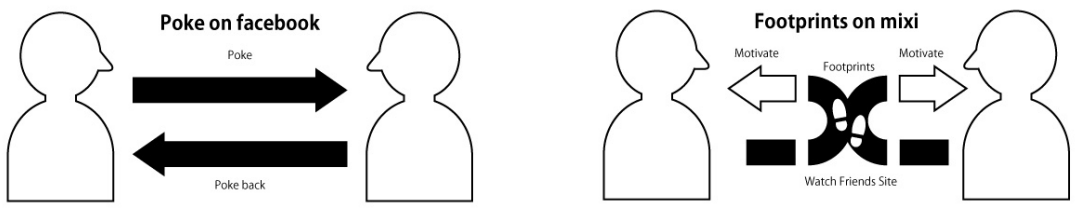

Fig. 12. Facebook and Mixi offer different ways to show a Friend's attention, as shown conceptually above. Facebook is direct with "poke." Mixi is subtle with "footprints".

Once again we see differences in persuasive design of the two SNS's. Facebook's "poking" is deliberate and direct. The system is designed to provoke users to return to Facebook and respond. Mixi has the same goal but it achieves return visits by enticement: the allure of seeing "footprints" evokes the target behavior.

\section{Conclusion and Implications}

In this paper we have compared how two social networking sites in different cultures motivate users toward the same four persuasive goals by using persuasion tactics. In general, the motivation elements in Facebook are bold and assertive. The Facebook service is designed to persuade users to take quick action with pointed outcomes. The Facebook system provides for direct and perhaps annoying notifications that serve as prompts to take action. By comparison, Mixi's approach to persuasion is subtle. The influence tactics are less direct and require more time to achieve results than those on Facebook. In general, Mixi users must invest more energy to start and maintain relationships through the service.

As researchers from the U.S. and Japan, each of us has experience working professionally in the other's culture. We bring that expertise to this comparative analysis. That said, in starting this research, we didn't expect - or want - to reach stereotypical conclusions about our cultures. As researchers we were open to finding that the Internet creates a new world, and that behaviors online may not reflect respective cultures. However, the evidence in our analysis clearly suggests that Facebook better reflects the persuasion dynamics that are common to US culture, while Mixi conveys a stronger Japanese sensibility.

Facebook and Mixi are among the first successful social networking services in any country. No matter what culture, we are at the beginning of learning how such services motivate and persuade people. The future will bring social networking 
services we can't imagine today. In this uncertainty, we believe that one constant remains: When new social networking services arrive, they will undoubtedly be designed to motivate and persuade users.

\section{References}

1. Fogg, B.J.: Persuasive Technology: Using Computers to Change What We Think and Do. Morgan-Kaufmann, San Francisco (2003)

2. Fogg, B.J., Eckles, D.: The Behavior Chain for Online Participation: How Successful Web Services Structure Persuasion. In: de Kort, Y., IJsselsteijn, W., Midden, C., Eggen, B., Fogg, B.J. (eds.) PERSUASIVE 2007. LNCS, vol. 4744, pp. 199-209. Springer, Heidelberg (2007)

3. Rosentstock, R., Strecher, V., Marshall, B.: Social Learning Theory and the Health Belief Model. Health Education Behavior 15(2), 174-183 (1988)

4. Bandura, A.: Self-efficacy: The exercise of control. W.H. Freeman, New York (1997)

5. Freedman, J.L., Fraser, S.C.: Compliance without pressure: The foot-in-the-door technique. Journal of Personality and Social Psychology 4, 195-202 (1966)

6. Tidwell, L.C., Walther, J.B.: Computer-mediated communication effects on disclosure, impressions, and interpersonal evaluations: Getting to know one another a bit at a time. Human Communication Research 28(3), 317-348 (2002)

7. Bandura, A.: Social foundations of thought and action: A social cognitive theory. PrenticeHall, Englewood Cliffs (1986)

8. Riegelsberger, J., Vasalou, A., Bonhard, P., Adams, A.: Reinventing trust, collaboration and compliance in social systems. In: CHI 2006 Extended Abstracts on Human Factors in Computing Systems CHI 2006, pp. 1687-1690. ACM, New York (2006)

9. Ellison, N.B., Steinfield, C., Lampe, C.: The benefits of Facebook "friends": Social capital and college students' use of online social network sites. Journal of Computer-Mediated Communication 12(4) (2007)

10. Markus, H.R., Mullally, P., Kitayama, S.: Selfways: Diversity in modes of cultural participation. In: Neisser, U., Jopling, D. (eds.) The conceptual self in context: Culture, experience, self-understanding, pp. 13-61. Cambridge University Press, Cambridge (1997)

11. Morling, B., Kitayama, S., Miyamoto, Y.: Cultural practices emphasize influence in the U.S. and adjustment in Japan. Personality and Social Psychology Bulletin 28, 311-323 (2002)

12. Khaled, R., Biddle, R., Noble, J., Barr, P., Fischer, R.: Culturally Aware Persuasive Technology: Collectivism Focused Strategies. In: Proceedings of the Seventh Australasian User Interface Conference (2006)

13. Baumeister, R.F., Leary, M.R.: The need to belong: Desire for interpersonal attachments as a fundamental human motivation. Psychological Bulletin 117, 497-529 (1995) 